\title{
émulations
}

\section{Martin Deleixhe - Aux bords de la démocratie. Contrôle des frontières et politique de l'hospitalité}

\section{Damien Simonneau}

Émulations - Revue de sciences sociales

2017, «Comptes rendus critiques, En ligne »

\section{Article disponible à l'adresse suivante}

https://ojs.uclouvain.be/index.php/emulations/article/view/7313

\section{Pour citer cet article}

Damien Simonneau, « Deleixhe Martin - Aux bords de la démocratie. Contrôle des frontières et politique de l'hospitalité », Émulations, en ligne. Mise en ligne le 18 septembre 2017.

DOI : 10.14428/emulations.cr.032

Distribution électronique : Université catholique de Louvain (Belgique) : ojs.uclouvain.be

(C) Cet article est mis à disposition selon les termes de la Licence Creative Commons Attribution, Pas d'Utilisation Commerciale 4.0 International. http://creativecommons.org/licenses/by-nc/4.0/

Éditeur : Émulations - Revue de sciences sociales / Presses universitaires de Louvain https://ojs.uclouvain.be/index.php/emulations

ISSN électronique : 1784-5734

UCL PRESSES

UNIVERSITAIRES 


\section{Martin Deleixhe - Aux bords de la démo- cratie. Contrôle des frontières et poli- tique de l'hospitalité}

Damien Simonneau ${ }^{1}$

Recensé : Deleixhe Martin (2016), Aux bords de la démocratie. Contrôle des frontières et politique de l'hospitalité, Paris, Classiques Garnier, coll. « PolitiqueS », 218 p.

"Comment penser l'ouverture des frontières dans un monde construit sur le postulat opposé ?» (Atak, Dumitru, 2015). Si en quelque sorte Martin Deleixhe n'apporte aucune réponse définitive à cette question, son ouvrage dresse un excellent panorama de philosophie politique pour appréhender la difficulté de penser cette utopie contemporaine, et plus encore de la mettre en œuvre.

Tout au long du livre, la frontière en démocratie apparaît comme une institution prise en tenaille entre deux principes : celui de la souveraineté populaire et celui du respect de normes universelles. La définition du demos, comme exigence d'autodétermination collective des peuples et comme geste souverain de désignation d'appartenance ou non à la communauté politique, peut-elle se faire sans empiéter sur la libre circulation des migrants et restreindre leurs droits fondamentaux, proclamés comme universels ? Tel est le dilemme qu'aborde l'ouvrage. Ce fil rouge s'étire sur trois parties indépendantes qui ne débouchent pas sur le développement d'une pensée philosophique originale de la question, mais plutôt sur un passage en revue des penseurs s'y confrontant.

Dans une première partie, l'auteur établit une typologie de quatre positions théoriques sur le contrôle démocratique des frontières. Ce dernier est pensé comme une exclusion arbitraire, raisonnée, immorale ou à résorber en fonction de la nature de la démocratie et de la conception de la frontière, souveraine, limite de justice, obstacle discriminatoire, ou chantier de la démocratie. Pour appréhender le dilemme initial, et faisant sien le postulat de l'absence de hiérarchisation entre autodétermination collective et adoption de normes universelles en démocratie, l'auteur indique sa préférence pour les penseurs de la quatrième catégorie où le contrôle des frontières est considéré comme une exclusion à résorber et où la frontière devient alors le « chantier de la démocratie» (Balibar, 2001). Dans la deuxième partie, il examine le droit cosmopolitique kantien et la morale de l'hospitalité qui pourraient réguler le contrôle des frontières

${ }^{1}$ Docteur en science politique, ATER à I'Université Paris 13, chercheur rattaché au CERAL et au Centre Émile Durkheim (Sciences Po Bordeaux). 
tout en concluant sur l'impossibilité d'appliquer une hospitalité inconditionnelle aux contingences étatiques. Dans la troisième partie, il réinjecte l'institution frontière pour envisager les contours de ce devoir d'hospitalité étatique avec Etienne Balibar. La frontière est alors densément saisie, d'une part, dans ses dimensions économiques internationales qui jugulent son ouverture ou non et, d'autre part, dans sa fonction de division sociale de la communauté politique sur fondement ethnique ou raciste. Reprenant Balibar, l'auteur émet les conditions de démocratisation de la frontière : élaborer une citoyenneté de résidence, lutter contre les discriminations à l'encontre des migrants, lutter contre les logiques d'exclusion du marché ou penser une formation sociale qui reconfigure la souveraineté étatique, comme l'a partiellement réalisé l'Union européenne. Cette démocratisation de la frontière implique la démocratisation de la démocratie elle-même.

Nous ne résumerons pas l'apport de chacun des philosophes au dilemme initial. Nous préférons retracer les trois étapes du raisonnement du livre, à savoir tout d'abord le dilemme posé par l'auteur, puis le panorama des différentes manières d'y répondre et enfin l'approfondissement des deux préférences théoriques de l'auteur. En effet, les conditions de mise en œuvre d'une hospitalité égalitaire sans rapport de domination et la démocratisation de l'institution frontière occupent la majeure partie du livre.

Le dilemme du contrôle frontalier démocratique

En démocratie, la frontière est le lieu d'un malaise. Elle est une « institution qui d'une part, délimite le territoire sur lequel s'applique la souveraineté d'un Etat démocratique, et d'autre part, départage les citoyens, membres à part entière de la communauté politique, des étrangers qui cherchent à y entrer ou des résidents qui y demeurent en étant sujets à ses lois sans pour autant participer à leur rédaction » (p. 27-28). Ce malaise est aigu car un régime démocratique se doit de justifier l'exclusion mise en place. Or, la légitimation de cette dernière ne peut ignorer l'universalité des droits fondamentaux des personnes migrantes. Ainsi, l'auteur porte à notre attention ce dilemme : la fonction de contrôle et d'expulsion ne peut échapper à la critique d'une violation du principe d'égalité morale des individus. Ce dilemme est accru pour toute démocratie en contexte de mondialisation où les politiques de sécurité frontalière et d'immigration en viennent à assigner à résidence les populations les plus démunies et à garantir la liberté de circulation aux élites mondialisées (Bauman, 2010 [1998] ; Carens, 1987). Les contrôles frontaliers vont donc à l'encontre d'une universalité du droit à émigrer (Withol de Wenden, 2013). L'exclusion semble ardue à justifier au regard des droits universels et exprime une part d'arbitraire inévitable dans la constitution du corps politique. Une fois ce dilemme exposé, l'ouvrage présente quatre positions théoriques pour l'appréhender.

Frontière souveraine, limite de justice, obstacle ou « chantier de la démocratie »

La typologie élaborée par l'auteur se fait en réponse à deux questions croisées. Premièrement : qui délibère sur la forme de la communauté politique ? (ce qui induit un 
spectre de formes démocratiques des plus autoritaires aux plus cosmopolites). Deuxièmement : comment se construit la légitimité de la frontière ? (de sa fermeture totale, à son ouverture totale voire son effacement).

Le premier profil de contrôle des frontières suit le schéma hobbesien, autour des travaux de Schmitt et d'Agamben notamment. La frontière est pensée comme un rempart face à l'hostilité extérieure. Son ouverture et sa fermeture relèvent donc d'une décision souveraine et arbitraire, tout comme les normes de l'Etat de droit qui ne sont jamais à l'abri de suspension en fonction des menaces invoquées. Le deuxième profil de contrôle des frontières se niche dans les thèses libérales de la souveraineté en convoquant Rawls ou Walzer. La frontière est alors une membrane sélective sur fondement humanitaire et utilitariste. Les critères d'admission au nom de la souveraineté populaire et des droits individuels tentent d'être réconciliés mais la souveraineté l'emporte puisqu'elle opère une distinction entre bon et mauvais migrant : «la frontière doit laisser passer les étrangers en situation de détresse ainsi que les étrangers qui rentrent dans les critères de justice (définis par les citoyens ou les nationaux) tout en maintenant les indésirables à l'extérieur » (p. 62). La position libérale vient in fine justifier un contrôle souverain de l'immigration au nom d'une conflictualité des intérêts de protection des migrants et ceux des citoyens les plus pauvres qui doivent bénéficier en priorité de la redistribution orchestrée au sein de la communauté politique. La troisième position théorique sur le contrôle des frontières est celle des cosmopolites libéraux et des partisans de la justice globale (Foessel, Lourme, 2016) prônant l'ouverture totale voire l'abolition des frontières. Les contrôles frontaliers sont pour les cosmopolites une trahison du projet universaliste démocratique, au nom de la liberté de circulation comme droit fondamental. Cette position radicale fait écho à une redéfinition de la souveraineté dispersée verticalement à différents niveaux de pouvoir autres que celui de l'Etat. Enfin, la quatrième position théorique est celle du contrôle des frontières comme chantier du politique. Conformément à Habermas ou encore à Benhabib, la souveraineté populaire et les droits de l'homme sont indissociables en démocratie. Ils ne peuvent être hiérarchisés et doivent être réconciliés. Cette réconciliation doit reposer sur un processus permanent de délibération sur les identités et les normes universelles de circulation dans le but de contraindre les gouvernements. Ceux-ci doivent par exemple aménager une transition entre droit au séjour, droit de résidence et accès à la citoyenneté. Pour Etienne Balibar, cette réconciliation passe plutôt par des épisodes de lutte par les « sans » pour les droits fondamentaux en se réappropriant la citoyenneté.

Ce panorama établi, l’auteur décide de s'inscrire « résolument dans la continuité de l'école qui postule une co-originarité agonistique des principes fondateurs de la démocratie libérale » (p. 93), soit la quatrième position en spécifiant les positions kantiennes et balibariennes.

Hospitalité, cosmopolitique et démocratisation de la frontière

Kant s'avère incontournable pour décrire les modalités d'accommodement entre souveraineté populaire et droits universels via le droit cosmopolitique. Celui-ci régule 
les relations d'un individu à l'Etat dont il n'est pas membre. Ce droit se décompose en un droit d'asile, un droit de visite auquel la souveraineté ne peut faire obstacle, et un droit de résidence qui lui, nécessite un consentement étatique souverain. Selon l'auteur, Kant en vient à réduire l'hospitalité à un droit de visite car le droit de résidence ainsi formulé maintient le privilège de la souveraineté étatique. Kant fait reposer son cosmopolitisme sur un progrès perpétuel, un idéal toujours inachevé. Il souligne donc l'écart impossible à combler entre un devoir moral inconditionnel (l'hospitalité) et sa réalisation qui nécessite de réaménager la souveraineté. Par exemple, les ressortissants européens ont obtenu un droit d'installation qui brouille la distinction entre visite/résidence mais son corollaire a été l'exclusion des étrangers extra-européens.

Martin Deleixhe fait alors un détour par les théoriciens de l'hospitalité de Derrida à Le Blanc (voir aussi Boudou, 2016). Pour le premier, celle-ci souffre d'une structure inégalitaire entre accueillant et accueilli qui soumet le second au premier. Pour Le Blanc, l'égalité de l'hospitalité est possible si l'hôte laisse venir l'étranger « dépourvu de préjugés » (p. 142). Ce détour permet de revenir à Balibar et aux conditions d'une hospitalité étatique (et non seulement interpersonnelle ou morale comme chez les deux auteurs précédents). Le philosophe marxiste veut faire tenir ensemble inclusion égalitaire de l'étranger et reconnaissance d'une primauté incontestable de la souveraineté populaire. Néanmoins, son raisonnement se heurte à la difficulté de proposer un schéma institutionnel ou de politiques publiques qui répondent complètement aux attentes de l'hospitalité démocratique.

Etienne Balibar apporte avant tout au dilemme initial une définition dense de la notion de frontière qui est décortiquée conformément à sa polysémie (elle n'a par exemple pas le même sens pour le clandestin ou l'homme d'affaires), son hétérogénéité via la myriade de dispositif de contrôle frontalier (Bigo, Guild, 2005) et sa surdétermination soit la prépondérance de facteurs historiques dans sa détermination. Il décortique enfin la frontière, bordure d'une formation sociale spécifique qui est actuellement celle de l'Etat-nation. Cette dissection s'opère selon la dimension économique de la frontière d'une part. Conformément à la pensée marxiste, ce sont les bourgeoisies qui à l'époque contemporaine se sont regroupées en unités politiques pour défendre une activité économique isolée par rapport aux concurrents internationaux en élaborant des politiques douanières. D'autre part, la frontière est disséquée selon sa dimension idéologique où elle devient un marqueur de la communauté imaginée nationale unifiant en une même unité politique des classes pourtant en lutte, dans un processus d'invention d'une ethnicité voire d'une race commune. La frontière va donc dans ce contexte mondialisé discriminer et stigmatiser l'immigré à cause de sa classe et de son ethnicité/race.

Cette définition dense étant proposée, Balibar entend réfléchir à la démocratisation de la frontière qui dans un contexte de mondialisation est le lieu où se propage le politique d'où le choix du titre de l'ouvrage « aux bords de la démocratie » (p. 172). Pour cela, il propose le concept d'« égaliberté » où liberté et égalité sont considérées comme indissociables en démocratie. La frontière est un obstacle à cela. Il est alors nécessaire de 
penser une « citoyenneté sans communauté » dont le principe d’inclusion/exclusion serait formulé en termes démocratiques. Pour Balibar, cette opération dépend des contextes. Elle peut prendre la forme de la lutte pour les droits politiques des travailleurs émigrés dans les années 1980, de celle pour le droit de vote des étrangers et de la lutte des sans-papiers par la suite. Dans chaque cas, il estime nécessaire d'ouvrir une négociation avec les étrangers pour établir une politique d'admission respectueuse, ce qui ouvre un débat annexe sur la représentation des migrants, des forums d'expression et des contraintes de ces délibérations sur les Etats (voir par exemple Pette 2014). Néanmoins ces actions ne font que démocratiser l'appartenance communautaire, mais ne résorbent pas l'exclusion territoriale... Le contenu positif d'une frontière réellement démocratisée n'en finit pas de questionner en fonction des conjonctures, ce qui la rend insaisissable...

En conclusion, Martin Deleixhe s'excuse de la déception (p. 186) des attentes du lecteur qui voudrait trouver un point d'ancrage solide pour allier les deux pans du dilemme du contrôle frontalier démocratique. Ce dilemme est connu, discuté mais insolvable surtout dans sa traduction en outils de politiques publiques légitimes. Par ce travail, l'auteur nous convainc néanmoins du nécessaire recours à l'imagination politique. Il nous amène aussi, en suivant l'intuition de Balibar, à porter notre attention sur l'agir politique des immigrés. En ce sens, ce panorama pédagogique de philosophie politique nous apparaît complémentaire de réflexions et d'actions « en chantier», tant théoriques que militantes, sur la mise en œuvre de la démocratisation de l'institution frontière. En effet, elles ambitionnent d'associer à l'élaboration des politiques migratoires la multiplicité des acteurs impliqués dans les migrations internationales, ou de mettre fin à la criminalisation et à l'enfermement des migrants, ou encore de stabiliser leur statut juridique et de lutter contre leur précarisation économique (Blanchard, Spire, 2012).

\section{Bibliographie}

Atak I., Dumitru S. (2015), "Pourquoi penser l'ouverture des frontières », Ethique publique, « Penser l'ouverture des frontières », vol. $17, \mathrm{n}^{\circ} 1$.

BALIBAR E. (2001), «Frontières du monde, frontières du politique », in Nous, Citoyens d’Europe ?, Les frontières, l'Etat, le peuple, Paris, La Découverte.

BAUMAN Z. (2010 [1998]), Le coût humain de la mondialisation, trad. Abensour, Alexandre, Paris, Fayard.

Bigo D., Guild E. (dir.) (2005), Controlling Frontiers. Free Movement Into and Within Europe, Burlington, Ashgate Publishing. 
BLANCHARD E., SPIRE A. (2012), « Remettre le droit des étrangers au centre des politiques d'immigration", Les sociologues s'invitent dans le débat, éditions du Croquant, Hors-série de la revue Savoir/Agir, p. 121-132.

Boudou B. (2016), Politique de l'hospitalité : une généalogie conceptuelle, Paris, CNRS Editions.

CAREnS J. H. (1987), «Aliens and Citizens. The Case for Open Borders", The Review of Politics, vol. 49, $n^{\circ}$ 2, p. 251-273.

Foessel M., Lourme L. (2016), Cosmopolitisme et démocratie, Paris, Puf, Vie des Idées.

Pette M. (2014), «Association: les nouveaux guichets de l'immigration? Du travail militant en préfecture », Sociologie, vol. 5, n 4, p. 405-421.

WiTHOL DE WeNDEN C. (2013), Le droit d'émigrer, Paris, CNRS éditions. 